\title{
Explaining the d-q Magnetic Couplings Theoretically in Saturated Smooth Air-Gap AC Machines
}

\author{
Mohamed Arbi Khlifi \\ Department of Electrical Engineering, University of Hail, Hail, Saudi Arabia \\ and SIME Laboratory, Ecole Nationale Superieure d' Ingenieurs (ENSIT), University of Tunis, Tunisia \\ mohamedarbi.khlifi@issatm.rnu.tn
}

\begin{abstract}
The current paper discusses the theoretically detailed physical analysis of the effects of main flux saturation in smooth air gap AC machines. For that purpose, three sections are presented. The first one deals for presenting equivalence saturation-air gap. The second presents the equivalence of inductances between saturation induction machines and a dismounted salient pole synchronous machine. The method analyzes the leakage inductances that properly represent the corresponding leakage fluxes. The third section treats the physical interpretation of the cross-saturation. It is possible to show that the equations and physical picture in a saturated smooth-air-gap machine are similar to the equations and physical picture in a non-saturated salient-pole machine, where mutual coupling exists between the direct and quadrature axes and the self-inductances are also different on the non-salient part of the machine due to the variation of the reluctance with the rotor angle.
\end{abstract}

Keywords-AC machines; physical interpretation; magnetic saturation

\section{INTRODUCTION}

In conventional models used in electrical engineering to represent electrical machines, it is quite common to neglect the magnetic saturation phenomenon, allowing the making of the simplifying assumption that inductances depend on the value of the flux imposed by currents, thanks to the non-linear relation: $\lambda_{m}\left(i_{m}\right)=L_{m}\left(i_{m}\right)$, where $\lambda_{m}\left(i_{m}\right)$ is a picture of the non-linear magnetization characteristic $B(H)$ of the employed ferromagnetic material [1-3]. Saturation is a phenomenon that becomes apparent when the current through an inductor exceeds a certain limit. In effect, saturation of an inductor occurs when the core of the inductor can no longer store magnetic energy [4]. An inductor is usually constructed using a ferromagnetic material such as iron or one of its alloys. A ferromagnetic material is composed of small domains whose dipole moments are randomly oriented. When the material is completely demagnetized, the net magnetic moment over the material is zero. As the current passes through the inductor, the domains in the material tend to align in the direction of the magnetic field intensity [5]. As more domains are aligned, the magnetic flux density increases. When all domains have aligned in the direction of the magnetic field intensity, the flux density reaches its saturation density and cannot increase further in the same fashion. As the magnetic field intensity increases beyond the saturation point, relatively small increases of the magnetic flux density occur. When a large number of magnetic domains align this way, the material is said to be in its saturation region [6]. Saturation representation is critical in the correct modeling of synchronous machines. The main effect of saturation in a synchronous generator is the decrease of its mutual inductances depending on the operating level of the generator. Such a decrease may be considerable as the generator is driven higher into saturation. Therefore, it is imperative that the effect of saturation is modeled in the parameter estimation procedure [7-12]. The representation of saturation is rather cumbersome because it is not possible to represent all the nonlinearities in a generator and the interaction between all circuits within the stator and the rotor (including the fictitious windings) with any single model for all the operating conditions. Therefore, a number of assumptions have to be made in order to enable satisfactory saturation modeling.

\section{PROBLEM FORMULATION}

The variation of the level of magnetic saturation sheets can be transformed and described by an air-gap with modulated thickness. Let us deal with the essential component of any revolving electric machine, in this occasion, the iron core coil. The usual structure is the one shown in Figure 1.

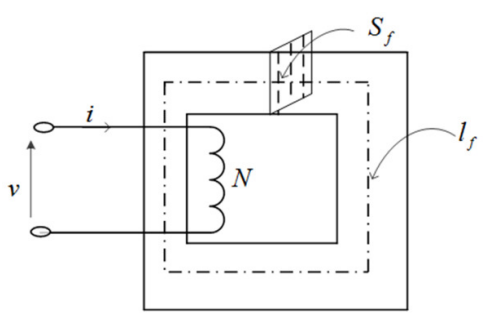

Fig. 1. Torus without air-gap.

The application of the law of Ampere gives:

$$
H_{f} l_{f}=N i
$$

In addition, the analysis between electric quantities and magnetic carries out (2):

$$
R_{f}=\frac{F}{\lambda_{f}}
$$

with

Corresponding author: Mohamed Arbi Khlifi 


$$
\begin{aligned}
& F=N i \\
& \lambda_{f}=B_{f} S_{f} \\
& B_{f}=\mu_{0} \mu_{r} H_{f}
\end{aligned}
$$

Finally, the magnetic reluctance of the iron which crosses the flux is:

$$
R_{f}=\frac{1}{\mu_{0} \mu_{r}} \frac{l_{f}}{S_{f}}
$$

In addition, one can admit that the same magnetizing force $F$ can produce the same flow $\lambda_{f}$ in the not homogeneous circuit of Figure 2 or iron is supposed to be perfect. The ring of the permanent magnet material and the air-gap are excited by a wire coil of $N$ turns and current $i$.

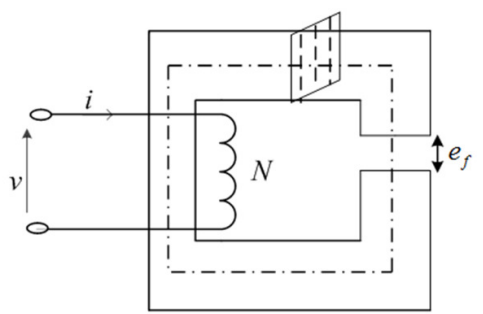

Fig. 2. Permanent magnet as a part of a magnetic circuit consisting of iron and an air gap, which is divided into an air-gap flux and leakage flux.

The geometry shown in Figure 2 is an excellent canonical problem to illustrate the salient features of permanent magnet circuits. Assume a small cross-section and ignore all leakage flux. The first topic is to show the steps necessary to determine $B$ in this problem, which is the same in the gap and the magnet because of the boundary condition. Under these conditions, the reluctances $R$ must be equal to their precedents $R_{f}$ described by (6). That is to say:

$$
R=R_{f}
$$

The new total reluctance is reduced, it is the one of the airgap $R_{\alpha}$ :

$$
R_{a}=\frac{1}{\mu_{0}} \frac{e_{f}}{S_{a}}
$$

Since the thickness of the air-gap is usually low, the useful section in the air and iron is unchanged:

$$
S_{f}=S_{\text {air }}=S
$$

The equivalence of (6) and (8) gives:

$$
e_{f}=\frac{l_{f}}{\mu_{r}}=\frac{C^{s t e}}{\mu_{r}}
$$

Thus, the thickness of the virtual air-gap $e_{f}$ is inversely proportional to the relative permeability of iron. When the magnetic medium is linear, $e_{f}$ corresponds with a minimal width. A rise in the level of saturation, by increase in current $I$ revolves a fall of $\mu_{r}$, synonymous with a widening of the airgap $e_{f}$, as it is indicated in Figure 3.

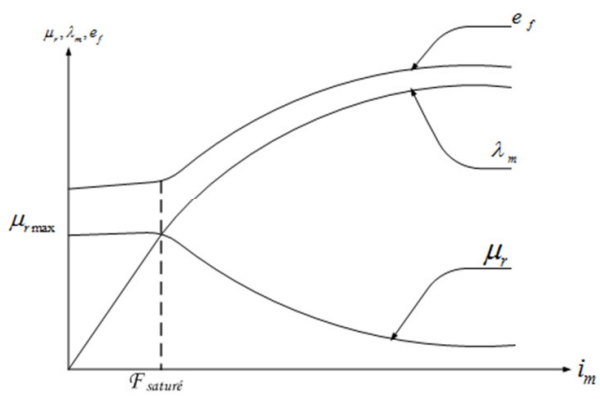

Fig. 3. Magnetizing curve, $\lambda_{m}, e_{f}, \mu_{r}$.

So for magnetic circuit containing a real air-gap, the resulting air-gap will be: $e+e_{f}$. In the electric machines the excitation $i$ and consequent induction vary sinusoidalement according to time (Figure 4 ).

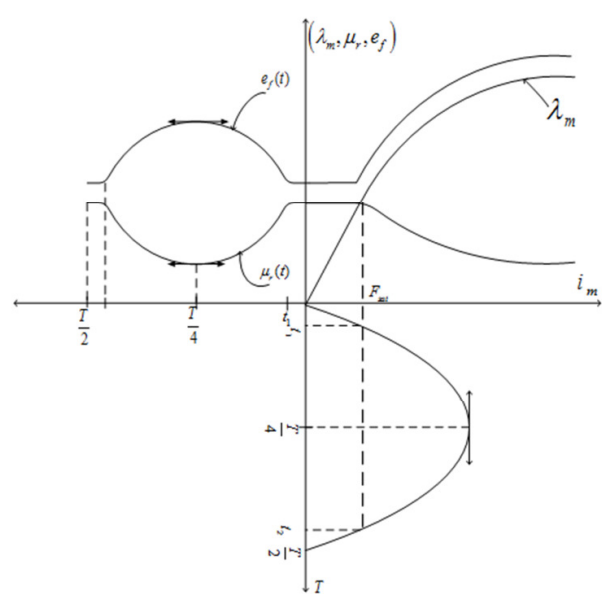

Fig. 4. Relation sheep between $\lambda_{m}, e_{f}, \mu_{r}$.

The main flux distribution around the air gap periphery is sinusoidal at any rate of saturation. Also the $\mathrm{mmfs}$ and voltages are sinusoidally distributed around the periphery. This assumption is equivalent to the supposition that the permeance of the iron is modeled by a factious variation of the air gap clearance (width). Since the real saturation effect caused by the iron core is to be replaced by supposition of a variable air gap clearance of the threshold of saturation the minimal permeability corresponds to maximum of the magnetomotrice force and the air-gap.

\section{EXPRESSIONS OF INDUCTANCES IN SALIENT POLE SYCHRONOUS MACHINES}

\section{A. Formulation of Voltage Equations}

In undamped three phase salient pole synchronous machines there are four mutually coupled coils: the three phase windings and the field. Therefore, the flux linkage equations for these windings result in the following matrix form: 


$$
[L]=\left[\begin{array}{cccc}
L_{a} & L_{a b} & L_{a c} & L_{a f} \\
L_{b a} & L_{b} & L_{b c} & L_{b f} \\
L_{c a} & L_{c b} & L_{c} & L_{c f} \\
L_{f a} & L_{f b} & L_{f c} & L_{f}
\end{array}\right]
$$

where $L_{j k}$ is a self-inductance when $j=k$ and a mutual inductance when $j \neq k$.The stator inductances and the coupled inductances between the stator and the rotor are time varying, while the rotor inductances are constants. Expressions for the inductances in (11) are given below.

\section{B. Inductances of the Stator Circuits}

The inductances can be assimilated with those of a diphase salient pole machine. The inductance of a diphase salient pole synchronous machine is expressed as:

$$
L_{a a^{\prime}}=L_{s m} \cos \beta-L_{s v} \cos (2 \theta-\beta)
$$

with

$$
\left\{\begin{array}{l}
L_{s m}=\frac{L_{\max }+L_{\min }}{2} \\
L_{s v}=\frac{L_{\max }-L_{\min }}{2} \\
\beta=\frac{\pi}{2}
\end{array}\right.
$$

\section{Stator Self-Inductances}

$$
\begin{aligned}
& L_{a}=L_{s m}-L_{s v} \cos (2 \theta) \\
& L_{b}=L_{s m}+L_{s v} \cos (2 \theta)
\end{aligned}
$$

$L_{s m}$ and $L_{s v}$ are constants and $L_{s m} \succ L_{s v}$. The double frequency term $(2 \theta)$ occurs due to the rotor saliency and the fact that self-inductances are the same for both rotor poles.

\section{Stator Mutual Inductances}

The stator to stator mutual inductances are a function of rotor position since they are influenced by rotor saliency.

$$
L_{a a^{\prime}}=-L_{s v} \sin (2 \theta)
$$

It is observed that (11) has time-varying terms which will cause complication when their derivatives are taken. Thus, it is convenient to refer all quantities to a rotor frame of reference through a Park's transformation.

\section{INTERPRETATION AND DISCUSSION}

\section{A. Interpretation}

Inductances can be comparable with those of a revolving machine diphase with salient poles whose principal inductances are $L_{m d y}$, according to the direct axis (weak air-gap, weak reluctance) and $L_{m}$ following the axis squaring. The standard mathematical model of an induction machine is given in terms of space vectors (underlined variables), in an arbitrary frame of reference $\omega_{\alpha}$ with the following set of equations:

$$
\begin{aligned}
& \bar{v}_{s}=R_{s} \bar{i}_{s}+\frac{d \bar{\lambda}_{s}}{d t}+j w_{a} \bar{\lambda}_{s} \\
& \bar{v}_{r}=R_{r} \bar{i}_{r}+\frac{d \bar{\lambda}_{r}}{d t}+j\left(\omega_{a}-w\right) \bar{\lambda}_{r}
\end{aligned}
$$

where $v, i$ and $\lambda$ denote voltage, current and flux linkage space vectors respectively, while indices $s$ and $r$ stand for stator and rotor, respectively. Equation of mechanical motion is irrelevant here and is hence omitted. $\omega$ is the electrical speed of rotation.

It is assumed that leakage flux saturation and main flux saturation can be treated independently. As only main flux saturation is discussed, leakage inductances are constants. Stator and rotor flux linkages are:

$$
\begin{aligned}
& \bar{\lambda}_{s}=l_{s} \bar{i}_{s}+\bar{\lambda}_{m} \\
& \bar{\lambda}_{r}=l_{r} \bar{i}_{r}+\bar{\lambda}_{m}
\end{aligned}
$$

where index $m$ denotes variables and parameters associated with magnetizing flux. The proposed common approach to introduce magnetic saturation in any $\mathrm{d}-\mathrm{q}$ existing model, for induction machine, relies only on the knowledge of the winding currents saturated model. For that reason, it will be first shown how to obtain it.

Leakage inductances in flux equation of induction machine (16)-(17) are assumed to be constant, only the main flux $\bar{\lambda}_{m}$ is subject to saturation. Deriving stator and rotor linkage fluxes, in (16)-(18), lead to the time derivative of the magnetizing flux $\bar{\lambda}_{m}$.

$$
\frac{d \bar{\lambda}_{s, r}}{d t}=l_{s, r} \frac{d \bar{i}_{s, r}}{d t}+\frac{d \bar{\lambda}_{m}}{d t}
$$

Therefore, $\frac{d \bar{\lambda}_{m}}{d t}$ has to be described by means of winding currents:

$$
\frac{d \bar{\lambda}_{m}}{d t}=\frac{d\left(\lambda_{m} e^{i \alpha}\right)}{d t}=e^{i \alpha}\left(\frac{d \lambda_{m}}{d t}+\lambda_{m} \frac{d \alpha}{d t}\right)
$$

where $\alpha$ is the angular position of $\bar{\lambda}_{m}$ with respect to the daxis. It also characterizes the position of the magnetizing current $\bar{i}_{m}$ in the air gap, since the hysteresis angle is neglected. Writing $\bar{i}_{m}, \alpha$ and $\frac{d \lambda_{m}}{d t}$ as:

$$
\begin{aligned}
& i_{m}=\sqrt{i_{d m}^{2}+i_{q m}^{2}} \\
& \alpha=\tan ^{-1} \frac{i_{q m}}{i_{d m}} \\
& \frac{d \lambda_{m}}{d t}=\frac{d \lambda_{m}}{d i_{m}} \frac{d i_{m}}{d t}=L_{m d y} \frac{d i_{m}}{d t}
\end{aligned}
$$

we get: 
$\frac{d \lambda_{d m}}{d t}=L_{d} \frac{d i_{d m}}{d t}+L_{d q} \frac{d i_{q m}}{d t}$

$\frac{d \lambda_{q m}}{d t}=L_{d q} \frac{d i_{d m}}{d t}+L_{q} \frac{d i_{q m}}{d t}$

$L_{m d y}=\frac{d \lambda_{m}}{d i_{m}}$ is called dynamic inductance by analogy with $L_{m}=\frac{\lambda_{m}}{i_{m}}$ static inductance. Both coefficients are given by the conventional nonlinear magnetization curve, determined experimentally.

$$
\begin{aligned}
& L_{d q}=\left(L_{m d y}-L_{m}\right) \cos \alpha \sin \alpha \\
& L_{d}=L_{m}+L_{d q} \cot \alpha \\
& L_{q}=L_{m}+L_{d q} \tan \alpha
\end{aligned}
$$

\section{B. Discussion}

Between rotor and stator we choose an integration path as shown in Figure 5. This path is defined by the angle $\theta$ and we can notice that due to symmetry, the flux density at the two airgap segments in the path is the same. If we assume the permeability of iron to be infinite, then $H_{\text {iron }}=0$. This means that for a given current $i$ in the coil, the flux density in the airgap varies sinusoidally with angle.

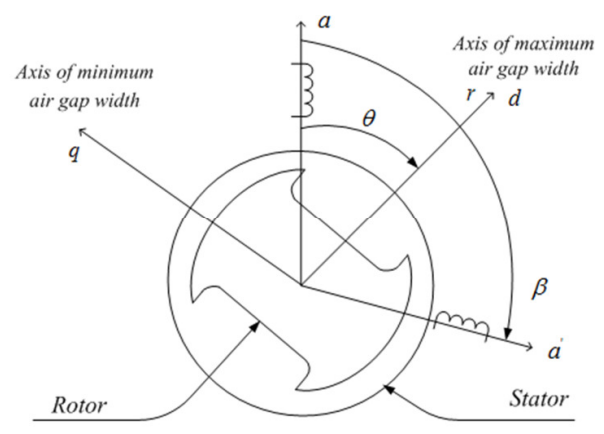

Fig. 5. Representation of salient pole synchronous machines.

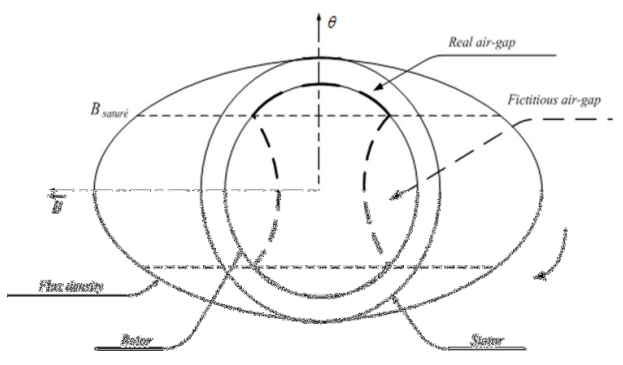

Fig. 6. A sinusoidal winding on the stator.

If the induction machine is saturated $L_{m d y} \prec L_{m}$ and the direct axis of the critical two phase machine coincides with the axis with maximum air gap width, that is the quadrature direction in conventional salient pole machines.

$$
L_{d q}=\left(L_{m d y}-L_{m}\right) \cos \alpha \sin \alpha=\left(\frac{L_{m d y}-L_{m}}{2}\right) \sin 2 \alpha
$$

where $\alpha$ is the angle position of the rotating magnetizing flux or current.

$$
\begin{aligned}
L_{d} & =L_{m}+L_{d q} \cot \alpha \\
& =L_{m}+\left(\frac{L_{m d y}-L_{m}}{2}\right) \frac{\cos \alpha}{\sin \alpha} \sin 2 \alpha \\
& =L_{m}+\left(L_{m d y}-L_{m}\right)\left(\frac{1+\cos 2 \alpha}{2}\right) \\
& =\left(\frac{L_{m d y}+L_{m}}{2}\right)-\left(\frac{L_{m d y}-L_{m}}{2}\right) \cos 2 \alpha \\
L_{q} & =L_{m}+L_{d q} \tan \alpha \\
& =L_{m}+\left(\frac{L_{m d y}-L_{m}}{2}\right) \frac{\sin \alpha}{\cos \alpha} \sin 2 \alpha \\
& =L_{m}+\left(L_{m d y}-L_{m}\right)\left(\frac{1-\cos 2 \alpha}{2}\right) \\
& =\left(\frac{L_{m d y}+L_{m}}{2}\right)+\left(\frac{L_{m d y}-L_{m}}{2}\right) \cos 2 \alpha
\end{aligned}
$$

For the saturated asynchronous machine static inductance is higher than that dynamic. While posing $\left(L_{m}=L_{\max }\right)$ and $\left(L_{m d y}=L_{\text {min }}\right)$, these inductances can be comparable with those of a diphase salient pole machine whose inductances along the direct axis, have weak air-gap and reluctance and inductances along the axis in squaring have strong reluctance and maximum of air-gap. For the saturated machine, permeance also depends in the state of saturation. The effect of saturation in the parts of iron results in the introduction of an equivalent air-gap with the same reluctance as the saturated parts. In the asynchronous machine, the function of permeance due to saturation varies along the armature. In fact, a saturated asynchronous machine can be regarded as equivalent to a diphase unsaturated salient pole synchronous machine.

We presented a concise method of determining transient phenomena in a capacitor-excited induction machine, which, of course, requires taking into account the main field saturation. The new feature in this paper is the incorporation of crossmagnetization effects in the analysis. By this, we added a useful application to a number of previous papers dealing with smooth-air-gap machines. The current applied model describes the induction machine quantities in d-q-components. An essential part of the model is the inductance matrix along with the definitions of its elements. It is true that recently some contradictory views on the phenomenon of cross-magnetization have been stated. Therefore it might be useful to repeat the basic considerations under which the present model holds: The magnetizing current produces a sinusoidal mmf distribution which gives rise to a flux-density curve containing a fundamental and saturation harmonics. The winding distribution is such that only the fundamental flux induces a voltage. Expressing the magnetizing current and the 
fundamental flux linkage as space-phasors, their magnitudes are related by the saturation curve. Note that there is a formal resemblance to the main inductances of a salient pole synchronous machine in stator frame representation, where the angular position of the pole axis is analog to the position $\alpha$ of the above mentioned magnetizing current space-phasor. In further consequence of this analogy, the (saturation dependent) $\left(L_{m d y}-L_{m}\right)$ takes the place of the difference between direct and quadrature axis synchronous inductances.

It has been shown that when the currents are used as statevariables, it is possible to show that the equations and physical picture in a saturated smooth-air-gap machine are similar to the equations and physical picture in a non-saturated salient-pole machine, where mutual coupling exists between the direct and quadrature axes and the self-inductances are different on the non-salient part of the machine due to the variation of the reluctance with the rotor angle. However, in contrast to this, in the saturated smooth-air-gap machine, the cross-saturation coupling and self-inductances vary with the angle of the magnetizing current. This is one of the reasons why a specific form of the inductance terms has been presented in $[1,2]$. This physical fact is also evidenced by (30)-(33), in contrast to the forms before where the small change of the magnetizing inductance is expressed by utilizing the difference between dynamic and static inductances. We utilized the first derivative of the magnetizing inductance with respect to the magnetizing current. The same derivative has been utilized in the paper under discussion, where an alternative formulation of these inductances has been given. If it is the purpose to focus attention to the analogy described above, or to give a clear analytical representation of the modifications in the impedance matrix, then it is useful to have expressions containing the angle of the magnetizing current space vector.

\section{CONCLUSION}

A linear analysis of the machine based on the coupled circuit theory and assuming sinusoidal distribution of both $\mathrm{mmf}$ and flux density is performed and the saturated model is derived by making some model parameters dependent on suitable saturation laws. In this paper, a comprehensive winding model of both induction motors and synchronous generators has been proposed where the effects of saturation are incorporated in the main flux path and sometimes attributed to the leakage reactance too.

Saturation of the magnetizing reactance must be driven by the main flux, whereas saturation of leakages is mainly dependent on the involved currents. In round rotor machines the influence of saturation only depends on the amplitude of the main flux, whereas in salient pole machines the flux angle may also be involved by considering, if known, different saturation characteristics as the flux moves from the direct to the quadrature axis. At steady state operation, only the main flux path is affected by saturation, whereas the leakages are usually maintained at their unsaturated values. According to the selected model, the saturated magnetizing reactance is defined as the slope of the chord corresponding to the operating point on the proper magnetizing characteristic. The effect of saturation at steady state is of main importance for a correct setting of the state variables before a transient analysis is started. For instance, the initial load angle of synchronous generators is closely related to the stability characteristic of the machines.

\author{
APPENDIX: LIST OF SYMBOLS
}

$N \quad$ Number of spire of magnetic circuit

I Current in magnetic circuit

$e_{f} \quad$ Length of the air-gap

$R_{f} \quad$ Magnetic Reluctance

$\lambda_{f} \quad$ Flux iron

$S_{f} \quad$ Surface of iron

$H_{f} \quad$ Magnetic excitation

$l_{f} \quad$ Length of the field lines

$B \quad$ Magnetic induction

$\mu_{r} \quad$ Relative permeability

$\mu_{0} \quad$ Permeability of vacuum

$d, q \quad$ Indices for direct and quadrature axis

$\bar{i} \quad$ Space vector winding current

\section{REFERENCES}

[1] M. A. Khlifi, H. Rehaoulia, "General modeling of saturated AC machines for industrial drives", COMPEL: The International Journal for Computation and Mathematics in Electrical and Electronic Engineering, Vol. 35, No. 1, pp. 44-63, 2016

[2] M. B. Slimene, M. A. Khlifi, M. B. Fredj, H. Rehaoulia, "Analysis of saturated self-excited dual stator induction generator for wind energy generation", Journal of Circuits, Systems and Computers, Vol. 24, No. 9, p. 1550129,2015

[3] M. B. Slimene, M. A. Khlifi, M. B. Ben, H. Rehaoulia, "Modeling of dual stator induction generator with and without magnetic saturation", Journal of Magnetics, Vol. 22, No. 5, pp. 145-153, 2015

[4] M. A. Khlifi, M. B. Slimene, M. B. Fredj, H. Rehaoulia, "Performance evaluation of self-excited DSIG as a stand-alone distributed energy resources", Electrical Engineering, Vol. 98, No. 2, pp. 159-167, 2016

[5] M. B. Slimene, M. A. Khlifi, M. B. Fredj, "Sensorless Speed Control for Dual Stator Induction Motor Drive Using IFOC Strategy with Magnetic Saturation”, Applied Computational Electromagnetics Society Journal, Vol. 32, No. 3, pp. 262-267, 2017

[6] I. Iglesias, L. Garcia-Tabares, J. Tamarit, "A D-Q model for the selfcommutated synchronous machine considering the effects of magnetic saturation", IEEE Transactions on Energy Conversion, Vol. 7, No. 4, pp. 768-776, 1992

[7] M. A. Khlifi, H. Rehaoulia "Modelling of salient pole synchronous machine with magnetic saturation" International Journal of Physical Sciences, Vol. 6, no. 21, pp. 4928-4932, 2011

[8] M. A. Khlifi, "Influence of Cross-Saturation on the Various Models of Induction Machine", International Journal of Automation and Control Engineering. Vol. 4, No. 4, pp. 268-272, 2016

[9] M. H. Shin, D. S. Hyun, S. B. Cho, S. Y. Choe, "An improved stator flux estimation for speed sensorless stator flux orientation control of induction motors", IEEE Transactions on Power Electronics, Vol. 15, No. 2, pp. 312-318, 2000

[10] L. Harnefors, "Design and analysis of general rotor-flux-oriented vector control systems", IEEE Transactions on Industrial electronics, Vol. 48, No. 2, pp. 383-390, 2001

[11] E. Levi, "A unified approach to main flux saturation modeling in D-Q axis models of induction machines", IEEE Transactions on Energy Conversion, Vol. 10, No. 3, pp. 455-461, 1995

[12] M. B. Slimene, M. A. Khlifi, "Performance Limits of Three-Phase SelfExcited Induction Generator (SEIG) as a Stand Alone DER", Journal of Electrical Engineering and Technology, Vol. 12, No. 1, pp. 145-150, 2017 